\title{
Recent Developments of $\zeta$-factor Microanalysis and Its Application to Armor Ceramics
}

Christopher Marvel $^{1}$, Kristopher Behler ${ }^{2}$, Vladislav Domnich ${ }^{3}$, Jerry LaSalvia ${ }^{4}$, Richard Haber ${ }^{5}$, Masashi Watanabe ${ }^{1}$ and Martin Harmer ${ }^{1}$

${ }^{1}$ Lehigh University, Bethlehem, Pennsylvania, United States, ${ }^{2}$ SURVICE Engineering; CCDC Army Research Laboratory, Aberdeen Proving Ground, Maryland, United States, ${ }^{3}$ Rutgers University; Ferro Corporation, King of Prussia, Pennsylvania, United States, ${ }^{4}$ CCDC Army Research Laboratory, Aberdeen Proving Ground, Maryland, United States, ${ }^{5}$ Rutgers University, Piscataway, New Jersey, United States

This work outlines several recent developments and applications of $\zeta$-factor microanalysis, a quantitative (scanning) transmission electron microscopy ((S)TEM) X-ray energy dispersive spectroscopy (XEDS) method that corrects for X-ray absorption, for compositionally characterizing armor-grade boron-based ceramics down to atomic-length scales [1].

Boron-based ceramics, in particular boron carbide, are used as armor ceramics because they exhibit low density $\left(\sim 2.52 \mathrm{~g} / \mathrm{cm}^{3}\right)$ and high hardness ( 40 GPa) [2]. Density and hardness are important properties because they reduce weight and maximize resistance to high-rate ballistic impacts, respectively [3]. Recent attempts to improve ballistic performance have been to strategically weaken grain boundaries to promote intergranular fracture, rather than transgranular fracture, thus increasing crack lengths and thereby improving fracture resistance [4]. Therefore, there is a need to accurately characterize boron carbide on fine length-scales to best understand, validate, and leverage interfacial process-structure-property relationships. Unfortunately, reliable compositional analysis of boron carbide, on any length-scale, is challenging. Conventional methods to analyze boron carbide (e.g. X-ray diffraction, Raman spectroscopy, combustion gas analysis) only reveal overall compositions without local composition fluctuations (e.g. at grain boundaries). Higher resolution analytical techniques in STEM (e.g. XEDS or electron energy loss spectroscopy (EELS)) are rather limited by X-ray absorption for XEDS, and sensitivity of background subtraction and the difficulties of signal detection of certain rare-earth elements for EELS which are common dopants used to improve performance of armor-grade ceramics. Overall, there was a need to develop a technique that can efficiently and accurately determine boron carbide bulk stoichiometry and grain boundary compositions.

This work extended $\zeta$-factor microanalysis to analyze boron carbide bulk stoichiometry and grain boundary composition. A collection of K-family $\zeta$-factors were determined using a NIST SRM-2063a thin film [5]. B K and $\mathrm{C} \mathrm{K} \zeta$-factors were independently determined using an extrapolation technique from wedged thin specimens of $\mathrm{SiB}_{6}$ and $\mathrm{SiC}$, respectively. Figure 1 a shows the $\zeta$-factors generated during this work where the open diamonds were directly determined from $\mathrm{SiB}_{6}$ and $\mathrm{SiC}$, the open circles were determined through the glass standard, and the closed circles were estimated from the determined $\zeta$ factors. Figure $1 \mathrm{~b}$ compares measurements of $\mathrm{B}$ concentration in $\mathrm{SiB} 6$ as a function of the specimen thickness with and without the absorption correction. The dashed line represents the nominal B concentration of stoichiometric $\mathrm{SiB}_{6}$. Upon correcting for absorption, the constant B concentration (i.e. independence of sample thickness) was considered validation that $\zeta$-factor microanalysis can accurately analyze boron-rich materials. Figure 1c shows the absorption factors that were applied for absorption corrections, exceeding $250 \%$ at sample thicknesses above $100 \mathrm{~nm}$. In addition, three bulk boron carbide 
specimens with different stoichiometries were analyzed and the measured compositions were validated with combustion gas measurements of the same bulk materials. Raster scan methods to determine grain boundary composition using $\zeta$-factor results were also extended based on prior techniques [6,7]. Overall, it was concluded that $\zeta$-factor quantification is a viable method to determine boron carbide stoichiometry and grain boundary composition.

An additional application, in which $\zeta$-factor quantification was central to the experimental capability, is to determine maximum $\mathrm{Si}$ solubility in boron carbide and average interfacial $\mathrm{Si}$ excess coverages as a function of bulk $\mathrm{Si}$ concentration within the polycrystalline diffusion zone of a $\mathrm{SiB}_{6} / \mathrm{B}_{4} \mathrm{C}$ diffusion couple heat treated at $1650{ }^{\circ} \mathrm{C}$ for 24 hours. The diffusion couple was used to determine maximum Si solubility in the boron carbide lattice and the consequential changes to $\mathrm{B} / \mathrm{C}$ stoichiometry and Si segregation behavior. Furthermore, if sufficient Si segregation was to occur, it was hypothesized that thick nanolayer complexions would form [8], thereby weakening grain boundaries and improving fracture resistance. Figure 2a shows a backscatter electron micrograph of the diffusion couple and the locations where thin specimens were extracted for $\zeta$-factor quantification. Figures $2 b$-d shows high-angle annular dark field STEM images where it was observed that the grain boundary structure at different points in the diffusion zone did not undergo much change, suggesting that a thick nanolayer complexion is not stable under these given processing conditions. However, as shown in Figure 2e, the average grain boundary composition (i.e. excess $\mathrm{Si}$ coverage in atoms $/ \mathrm{nm}^{2}$ ) was measured to increase with increasing bulk Si concentration in the diffusion zone, thus suggesting that while a structural complexion transition did not occur, there was indeed a wide variability in grain boundary composition. This result supports the notion that grain boundaries could be relatively weaker with increasing Si coverage. Overall, the maximum Si solubility in the matrix was measured as 1.7 at.\%, corresponding with a $5.6 \mathrm{~B} / \mathrm{C}$ ratio, and the maximum $\mathrm{Si}$ excess coverage was 8.7 atoms $/ \mathrm{nm}^{2}$. Notably, considering the nearly constant increase of Si excess coverage against $\mathrm{Si}$ concentration in the bulk, it was determined that the grain boundary segregation behavior agrees with the Gibbsian absorption definition where a higher bulk solubility leads to an increase of grain boundary segregation [9].
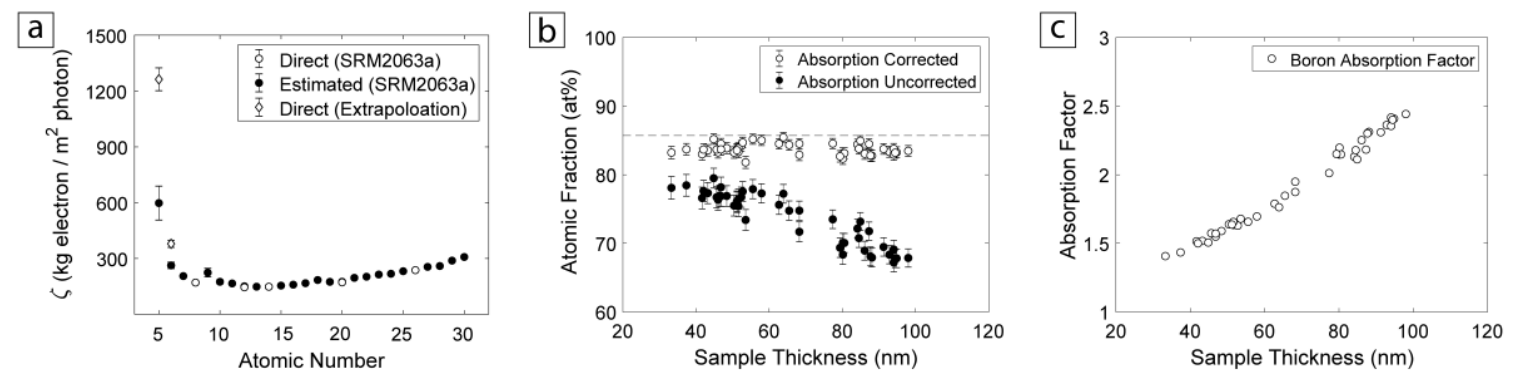

Figure 1. Summary of (a) $\zeta$-factors used throughout this study, (b) B concentration vs sample thickness measured from a $\mathrm{SiB} 6$ standard which was used as validation, and (c) a summary of the associated absorption correction factors applied to the B K-line X-rays where a $250 \%$ correction was applied at a sample thickness of $100 \mathrm{~nm}$. 

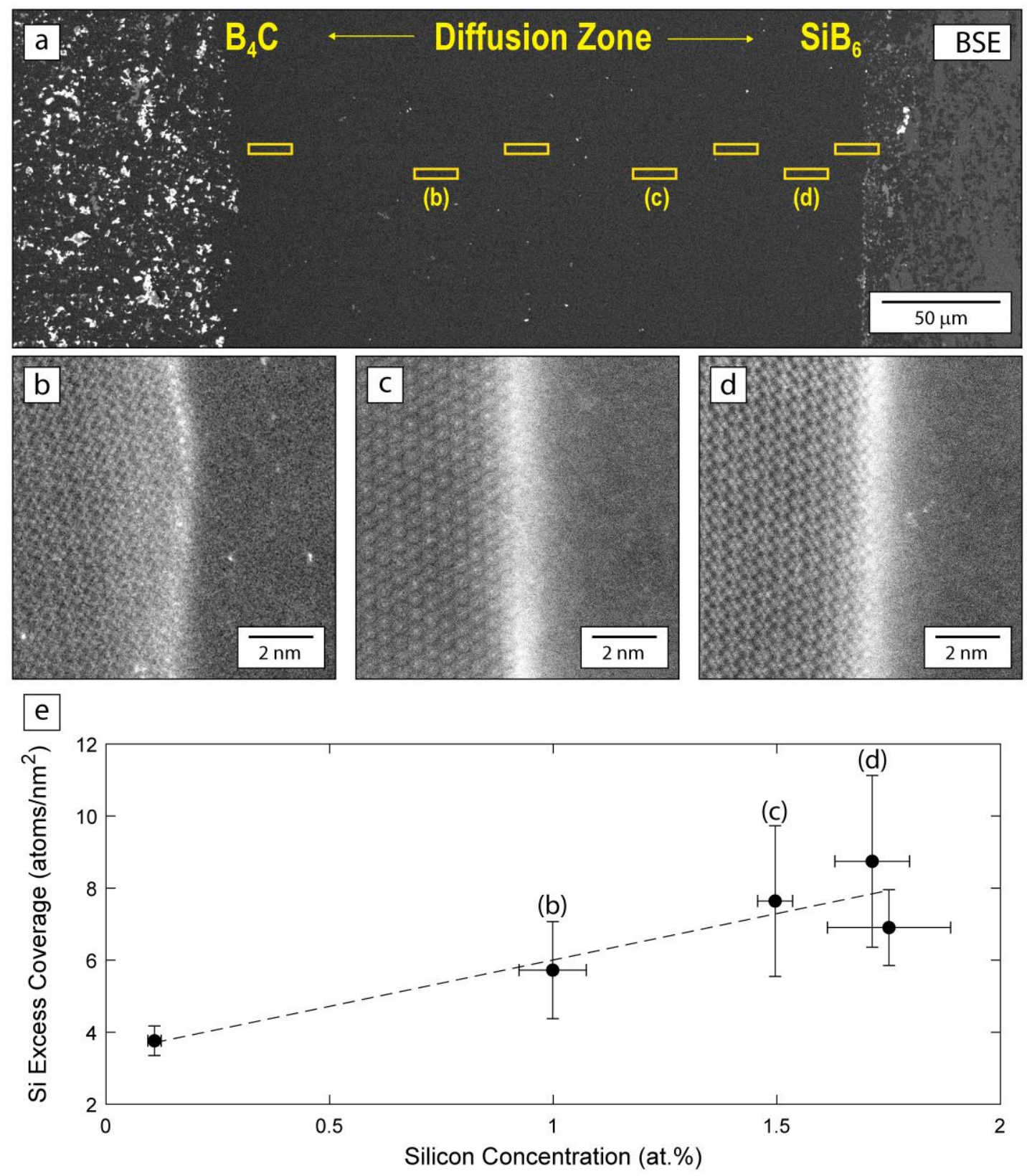

Figure 2. Summary of analytical results from the (a) B4C:SiB6 diffusion couple where multiple thin specimens (shown in yellow) were extracted for $\zeta$-microanalysis , (b-d) high-angle annular dark field micrographs showing minimal differences in grain boundary structure from different regions of the diffusion zone, and (e) grain boundary segregation dependence of Si concentration in the bulk boron carbide lattice.

\section{References}

[1] C.J. Marvel, K. D. Behler, J. C. LaSalvia, V. Domnich, R. A. Haber, M. Watanabe, and M. P. Harmer. "Extending $\zeta$-factor microanalysis to boron-rich ceramics: Quantification of bulk stoichiometry and grain boundary composition." Ultramicroscopy 202 (2019): 163-172.

[2] V. Domnich, S. Reynaud, R.A. Haber, M. Chhowalla. "Boron carbide: structure, properties, and stability under stress." Journal of the American Ceramic Society 94, no. 11 (2011): 3605-3628. 
[3] J.C. LaSalvia, J. Campbell, J. J. Swab, and J. W. McCauley. "Beyond hardness: ceramics and ceramicbased composites for protection." Jom 62, no. 1 (2010): 16-23.

[4] K.D. Behler, C.J. Marvel, J.C. LaSalvia, S.D. Walck, and M.P. Harmer. "Observations of grain boundary chemistry variations in a boron carbide processed with oxide additives." Scripta Materialia 142 (2018): 106-110.

[5] M. Watanabe and D. B. Williams. "The quantitative analysis of thin specimens: a review of progress from the Cliff-Lorimer to the new $\zeta$-factor methods." Journal of microscopy 221, no. 2 (2006): 89-109. [6] U. Alber, H. Müllejans, and M. Rühle. "Improved quantification of grain boundary segregation by EDS in a dedicated STEM." Ultramicroscopy 69, no. 2 (1997): 105-116.

[7] V.J. Keast and D. B. Williams. "Quantification of boundary segregation in the analytical electron microscope." Journal of microscopy 199, no. 1 (2000): 45-55.

[8] A.R. Krause, P.R. Cantwell, C.J. Marvel, C. Compson, J.M. Rickman, and M.P. Harmer. "Review of grain boundary complexion engineering: Know your boundaries." Journal of the American Ceramic Society 102, no. 2 (2019): 778-800.

[9] J.W. Gibbs. The collected works of J. Willard Gibbs. No. 536.7092. Yale Univ. Press, 1948. 